\title{
Conserved Microsynteny of NPR1 with Genes Encoding a Signal Calmodulin-Binding Protein and a CK1-Class Protein Kinase in Beta vulgaris and Two Other Eudicots
}

\author{
David Kuykendall, Jonathan Shao, and Tammy Murphy \\ Molecular Plant Pathology Laboratory, Agricultural Research Service, United States Department of Agriculture, \\ 10300 Baltimore Avenue, Building 004, Room 120, Beltsville, MD 20705, USA
}

Correspondence should be addressed to David Kuykendall, david.kuykendall@ars.usda.gov

Received 8 January 2008; Accepted 19 August 2008

Recommended by Silvana Grandillo

\begin{abstract}
NPR1 is a gene of central importance in enabling plants to resist microbial attack. Therefore, knowledge of nearby genes is important for genome analysis and possibly for improving disease resistance. In this study, systematic DNA sequence analysis, gene annotation, and protein BLASTs were performed to determine genes near the NPR1 gene in Beta vulgaris L., Medicago truncatula Gaertn, and Populus trichocarpa Torr. \& Gray, and to access predicted function. Microsynteny was discovered for NPR1 with genes $C a M P$, encoding a chloroplast-targeted signal calmodulin-binding protein, and CK1PK, a CK1-class protein kinase. Conserved microsynteny of NPR1, CaMP, and CK1PK in three diverse species of eudicots suggests maintenance during evolution by positive selection for close proximity. Perhaps close physical linkage contributes to coordinated expression of these particular genes that may control critically important processes including nuclear events and signal transduction.
\end{abstract}

Copyright (C) 2008 David Kuykendall et al. This is an open access article distributed under the Creative Commons Attribution License, which permits unrestricted use, distribution, and reproduction in any medium, provided the original work is properly cited.

\section{INTRODUCTION}

Research done on Arabidopsis thaliana (L.) Heynth over a 10 -year period in a number of laboratories has amassed considerable evidence that the NPR1 gene (also called NIM1) is of central importance in determining the plant ability to resist microbial attack [1]. In essence, global plant defense responses to pathogen invasion are controlled by the NPR 1 gene product and intracellular redox state, since an inactive dimeric NPR1 protein in the cytosol is reduced to the active monomer which then migrates to the nucleus and activates expression of pathogen-induced "pathogenesis-related" (PR) genes [2].

The central role of NPR1 in positively activating defense mechanisms in response to biotic stress suggests the possibility of enhancing disease resistance in plants by genetic manipulation of the NPR1 gene. In fact, an increasing number of attempts to improve disease resistance in plants by modifying expression of NPR1 have been reported [3-7].

Microsynteny is genomics information that can be used to predict the location of homologous genes in different species. Knowledge of microsynteny of genes colinear with NPR1 in crop species could perhaps be used to devise innovative strategies for molecular genetic modification in order to improve disease resistance. As a step towards identifying genes located near the NPR1 gene in sugarbeet, a bacterial artificial chromosome (BAC) library [8] was screened and an NPR1-carrying clone, SBA091H24, was identified [9]. The $B$. vulgaris BvNPR1 gene encodes a predicted protein product being $100 \%$ identical to that deduced from the sequence of the cDNA for B. vulgaris NPR1 (GenBank accession AY640381). SMART analysis of the predicted BvNPR1 gene product [9] showed a BTB/POZ domain and two ARDs, or ankyrin repeat domains [10], both being characteristic of NPR1 proteins and other transcriptional activators within the nucleus. NPR1 is responsible for disease resistance priming or "induced resistance," a result of coordinated expression of multiple defense mechanisms/pathways to effectively resist microbial attack [11].

The NPR1 gene in sugarbeet has been only recently shown [12] to be essential for induced systemic resistance, as in the model A. thaliana (L.) Heynth [1]. In both 
plant species, the activated form of NPR1 migrates into the nucleus and activates the transcription of genes involved with resisting disease-forming microbes [12].

Conservation of microsynteny among distinct families of eudicots was discovered in Lycopersicon esculentum Mill. (tomato) and A. thaliana, where large-scale duplications followed by selective gene loss have created a network of chromosomal synteny [13] —an accepted paradigm. By developing physical genetic maps based on expressed sequence tags (ESTs), Dominguez et al. [14] discovered conserved synteny with Arabidopsis among the genomes of four phylogenetically divergent eudicot crops, namely, sugarbeet, potato, sunflower, and plum.

In this study, complete BAC sequence analysis identified two core plant genes tightly physically linked to NPR1, and established a conservation of microsynteny between the NPR1 gene regions of sugarbeet and two other eudicot species. We report the gene content and organization of a $130 \mathrm{~Kb}$ DNA contig (continuous fragment) from an NPR1carrying sugarbeet BAC. Comparison of similar NPR1carrying DNA contigs from $M$. truncatula and P. trichocarpa showed that orthologs of genes encoding NPR1, a signalpeptide calmodulin-binding protein (CaMP) and a CK1class dual-specificity protein kinase (CK1PK) occur in the same order and with a conserved direction of transcription in three divergent species of eudicots. This suggests positive natural selection for maintaining the physical proximity of genes whose products control certain essential nuclear events and a particular signal transduction function, as yet undefined.

\section{MATERIALS AND METHODS}

\subsection{DNA sequencing}

Genomic DNA of B. vulgaris hybrid US H20 [15], with an estimated $750 \mathrm{Mb}$ genome size, had previously been used to construct a BAC library by ligating large DNA fragments resulting from partial HindIII digestion into vector pECBAC1 [16]. About 34,500 clones comprised the BAC library, and the average insert size was about $120 \mathrm{~Kb}$, providing about 6.1X genome coverage [8]. Primers designed on the basis of data extracted from GenBank accession AY640381, a cDNA sequence for B. vulgaris NPR1, were utilized to screen and identify a $B v N P R 1$-carrying BAC [9]. The presence of a complete genomic $B v N P R 1$ gene was established by partial DNA sequence analysis of BAC clone SBA091H24 (GenBank accession DQ851167) [9].

BAC sequencing was completed at Washington University's Genome Sequencing Center in St. Louis, Mo, USA (http://genome.wustl.edu/). The BAC clone SBA091H24 was provided to the Genome Sequencing Center as a glycerol stock. Purification, library construction, shotgun cloning, and sequence analysis were performed on a sufficient number of random subclones to provide about $9.4 \mathrm{X}$ coverage. ABI 3730 capillary sequencers were used. Data was assembled using the phred/phrap suite (http://www.phrap.org/).

\subsection{Gene annotation}

Analysis of the sequence data was performed using Lasergene (DNASTAR, Inc., Madison, WI, USA) for assembly and NCBI BLAST [17]. The sequence contig was screened for coding sequence using a combination of the following programs: GeneMark [18, 19] for eukaryotes (http://exon.gatech.edu/GeneMark/eukhmm.cgi), GenScan (http://genes.mit.edu/GENSCAN.html), FGENESH (http:// softberry.com/), and GRAIL (http:/grail.lsd.ornl.gov/grail$\exp /)$. In all cases, A. thaliana was chosen as a model, and default settings were used. BLASTP searches were performed at the National Center for Biotechnology Information (NCBI) website (http://www.ncbi.nlm.nih.gov/BLAST/). Percent identities and percent similarities were obtained using BLAST alignments. Manual curation of proteins was performed using Lasergene MegAlign and EditSeq sequence analysis software, where applicable simple modular architecture research tool (SMART) [20] database (http://smart.emblheidelberg.de/) and Motif Scan (http:// myhits.isb-sib.ch/cgi-bin/motif_scan) were used to identify protein domains and motifs, respectively. ARTEMIS (http:// www.sanger.ac.uk/Software/Artemis/) was used to collate data and facilitate annotation.

Calmodulin target database (http://calcium.uhnres.utoronto.ca/ctdb/ctdb/home.html) was used to identify the most likely calmodulin-binding site in a peptide sequence. The hmmtop server (http://www.enzim.hu/hmmtop/) was used to predict transmembrane helices, and a signal peptide was detected using the SignalP 3.0 and TargetP 1.0 servers (http://www.cbs.dtu.dk/services/).

BAC chromosome and genomic annotation datasets for M. truncatula and O. sativa L. were accessed through TIGR (http://www.tigr.org/). P. trichocarpa (black cottonwood) genome information [21] was accessed through NCBI (http:// www.ncbi.nlm.nih.gov/entrez/query.fcgi? db=genomeprj\& cmd=Retrieve\&dopt=Overview\&list_uids=10770).

\subsection{Identification of colinearity}

BLAST searches were performed for protein products of the predicted ORFs of the B. vulgaris NPR1 BAC against databases for A. thaliana, M. truncatula, P. trichocarpa, and O. sativa L. ssp. japonica. High-scoring pairs (HSPs), the predicted protein products with highly significant matches, were considered as products of orthologous genes. Corresponding DNA regions are considered to be microcolinear when two or more orthologous genes are present in physical proximity, in the same order, and are transcribed in the same direction.

GenBank accession NC_008472.1 is representative of linkage group VI of $P$. trichocarpa [21], and the subsets of particular interest were coordinates from 8404895 to 8453314. GenBank accession AC124609 represents BAC clone mth229b13, a subset of chromosome 2 of $M$. truncatula (http://www.tigr.org/tigr-scripts/medicago/contig_location_ association.pl?chromosome $=2) \quad[22,23]$. AC124609 (1... 61740 bases) was extracted and compared to our B. vulgaris GenBank accession EF101866 (1...129695 bases). 


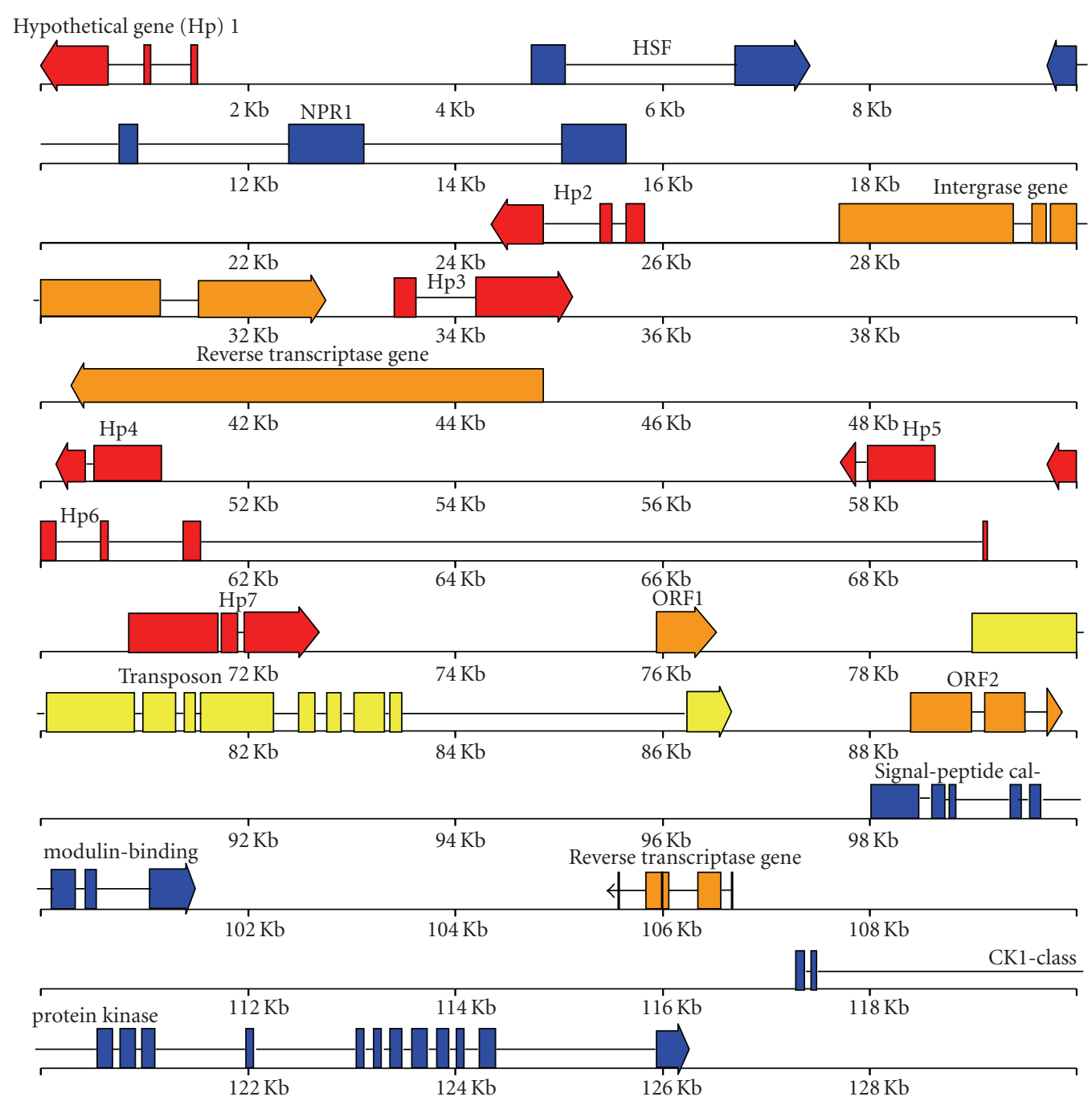

FIGURE 1: Schematic representation of genes annotated on the $130 \mathrm{~Kb}$ genomic NPR1-carrying BAC from sugarbeet (GenBank accession EF101866) based on FGENESH, GenScan, GeneMark, and GRAIL gene finders as well as SMART and Motif Peptide Scan results. (Blue: core plant genes involved with nuclear events/signal transduction/defense; yellow: transposon; orange: retrotransposons; red: encode only hypothetical or unknown proteins). The predicted genes begin with a bar and end with an arrowhead, thus indicating the direction of transcription.

\section{RESULTS}

A $130 \mathrm{~Kb}$ BAC designated SBA091H24, containing B. vulgaris chromosomal DNA, more specifically the sugarbeet NPR1 gene [9], was sequenced and fully annotated (GenBank accession EF101866). The bioinformatics tools FGENESH, GeneMark, GenScan, and GRAIL were used as gene finders. Predicted gene names and predicted functions of deduced amino acid sequences, where possible, are presented in Table 1, and a visual representation of exon structure is shown in Figure 1 . On the $130 \mathrm{~Kb}$ contig, a total of 17 potential open reading frames (ORFs), or protein coding regions, were identified. Only four out of the 17 open reading frames (ORFs) were predicted to produce protein products with high amino acid similarity to known products of core plant genes (Table 1). In addition to four core plant genes, the $130 \mathrm{~Kb}$ contig contains five retrotransposon genes, a transposon gene, and seven other genes whose products lacked a predicted function.

In addition to NPR1, another core plant gene, predicted on the $130 \mathrm{~Kb}$ sugarbeet DNA contig from BAC clone SBA091H24, was composed of two exons that encode a heat shock factor (HSF) protein with a conserved DNA binding domain $\left(E=2 \mathrm{e}^{-29}\right)$ from amino acid positions 45 to 205 . This HSF gene is located between the NPR1 gene and gene $H p 1$, encoding a hypothetical protein with some similarity to retrotransposon-encoded proteins. The HSF gene and NPR1 are transcribed in opposing directions. The predicted HSF protein has moderately high similarity (Table 1) to the protein product of HSFA9-a leaf pattern morphogenesiscontrolling gene of sunflower, Helianthus annuus [24].

Beginning at about $70 \mathrm{~Kb}$ upstream of NPR1, another core plant gene encodes a calmodulin-binding protein (CaMP) that, as SMART analysis revealed, has an IQ domain 
TABLe 1: Predicted genes and their designated functions.

\begin{tabular}{|c|c|c|c|c|c|}
\hline Gene & Product length (a.a.) & Best BLAST Hit ${ }^{\mathrm{a}}$ & $E$-value & Similarity & Designation $^{\mathrm{b}}$ \\
\hline Hp1 & 259 & ABE85118 & $5.0 E-08$ & $49 / 101$ & Hypothetical $^{c}$ \\
\hline $\begin{array}{l}\text { HSF (similar to } \\
\text { HSFA9) }\end{array}$ & 337 & AAM43804 & $3.4 E-47$ & $185 / 320$ & $\begin{array}{l}\text { HSF transcription } \\
\text { factor }\end{array}$ \\
\hline NPR1 & 604 & AAT57640 & $0.0 E+00$ & $604 / 604$ & $\begin{array}{l}\text { NPR1 disease } \\
\text { resistance }\end{array}$ \\
\hline $\mathrm{Hp} 2$ & 259 & none & & & Hypothetical $^{c}$ \\
\hline Integrase & 1516 & ABE91091 & $0.0 E+00$ & $903 / 1535$ & Integrase (copia type) \\
\hline $\mathrm{Hp} 3$ & 403 & ABD83280 & $1.1 E-124$ & $315 / 325$ & Unknown $^{\mathrm{d}}$ \\
\hline $\begin{array}{l}\text { Reverse transcriptase } \\
\text { (RT) }\end{array}$ & 1501 & ABE83303 & $0.0 E+00$ & $996 / 1503$ & $\begin{array}{l}\text { Gypsy-type } \\
\text { retrotransposon }\end{array}$ \\
\hline $\mathrm{Hp} 4$ & 302 & CAH67120 & $1.0 E-13$ & 78/177 & Hypothetical $^{\mathrm{c}}$ \\
\hline Hp5 & 263 & ABD83301 & $1.0 E-09$ & $41 / 63$ & Hypothetical $^{\mathrm{c}}$ \\
\hline Hp6 & 222 & none & & & Hypothetical $^{\mathrm{c}}$ \\
\hline $\mathrm{Hp} 7$ & 593 & ABD83280 & $4.1 E-60$ & $186 / 282$ & Unknown $^{\mathrm{d}}$ \\
\hline $\begin{array}{l}\text { ORF1 of Coe1 } \\
\text { (a composite of class I } \\
\text { and class } 2 \text { elements) } \\
{[25]}\end{array}$ & 188 & NP_199616.1 & $1 e-17$ & $106 / 192$ & $\begin{array}{l}\text { Hypothetical } \\
\text { (retroelement-like } \\
\text { gene) }\end{array}$ \\
\hline $\begin{array}{l}\text { Coe1 (transposon } \\
\text { gene) }\end{array}$ & 1399 & ABE82848 & $0.0 E-00$ & $604 / 956$ & Transposon \\
\hline $\begin{array}{l}\text { ORF2 of Coe1 (an } \\
\text { integrase pseudogene) }\end{array}$ & 297 & ABA95677 & $4.0 E-70$ & $177 / 298$ & $\begin{array}{l}R v t 2 \text { domain } \\
\text { integrase pseudogene }\end{array}$ \\
\hline $\begin{array}{l}\text { Calmodulin-binding } \\
\text { protein (CaMP) }\end{array}$ & 525 & NP_974673 & $7.0 E-161$ & $361 / 495$ & $\begin{array}{l}\text { Signal-peptide } \\
\text { calmodulin-binding } \\
\text { protein }\end{array}$ \\
\hline $\begin{array}{l}\text { RT (reverse } \\
\text { transcriptase) }\end{array}$ & 198 & ABF81417 & $3.0 E-37$ & $126 / 213$ & RT-like gene \\
\hline $\begin{array}{l}\text { CK1-class protein } \\
\text { kinase }(\mathrm{CK} 1 \mathrm{PK})\end{array}$ & 473 & BAB92346 & $0.0 E+00$ & $411 / 476$ & $\begin{array}{l}\text { CK1-class } \\
\text { dual-specificity } \\
\text { protein kinase }\end{array}$ \\
\hline
\end{tabular}

${ }^{a}$ GenBank accession number or protein ID of the best BLAST hit, followed by the E-value and percent (similar/total amino acids) similarity between the query and the best hit.

${ }^{b}$ Designation based on the deductions possible via bioinformatics tools listed in Section 2. Functional classification based on the result of protein BLAST search.

${ }^{\mathrm{c}} \mathrm{NA}$ : not applicable; putative function of the product was not identified.

${ }^{\mathrm{d}}$ Similar to FGENESH 21 [26].

from amino acid positions 131 to 153 . Motif Scan suggested (1) an IMP dehydrogenase/GMP reductase domain at amino acids $121-444,(2)$ several protein kinase phosphorylation sites, and (3) an involucrin repeat at amino acids 206215. The predicted protein product gave numerous BLAST hits to calmodulin-binding proteins. Calmodulin target database predicted amino acid positions 201-209 as the most likely calmodulin-binding site. Transmembrane helices were predicted at amino acid positions 337-353 by the hmmtop server, and the SignalP 3.0 server predicted a cleavage site in the N-terminal signal-peptide sequence between glutamine and lysine in amino acid positions 17 and 18. Thus, we deduced that the mature CaMP protein is 508 amino acids in length. The pro-CaMP was predicted as 525 amino acids in length, and the presence of a membrane-targeted $\mathrm{N}$-terminal signal-peptide sequence suggests that peptide maturation occurs upon passage into or through a membrane. TargetP predicted that the targeted sites of subcellular localization of mature protein were chloroplasts.

Beginning at about $90 \mathrm{~Kb}$ upstream of NPR1, a third core plant gene encodes a "casein kinase class 1" protein kinase (CK1PK), identified by numerous BLAST hits with $E$-values of 0 to CK1PKs. SMART analysis revealed a dual-specificity STYKc protein kinase domain from amino acids 9-211. This type of kinase phosphorylates either serine, threonine, or tyrosine residues.

Conservation of microsynteny was discovered in $B$. vulgaris, M. truncatula, and P. trichocarpa (Figure 2), but not in A. thaliana or O. sativa L., by comparative DNA analysis of three core plant genes: (1) a CK1 class dual-specificity protein kinase gene, (2) a signal calmodulin-binding protein gene, and (3) the disease resistance-priming NPR1 gene. The high degree of amino acid similarity as well as identity of the predicted products of these respective microcolinear genes 


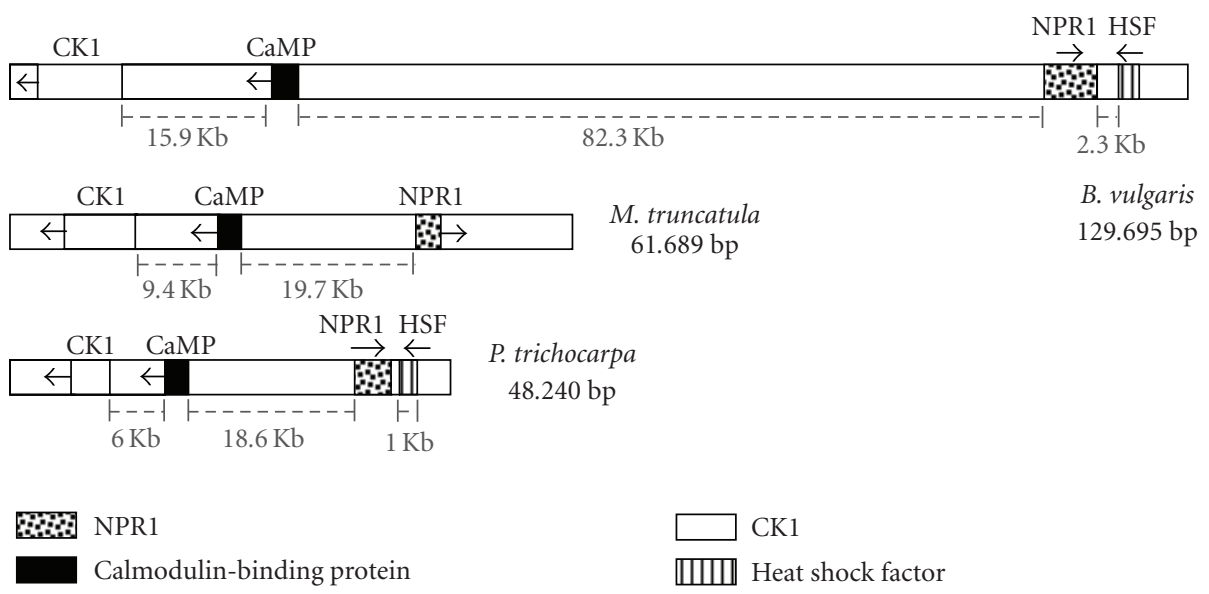

FIGURE 2: Schematic representation of the microcolinearity found between NPR1-carrying regions of (a) B. vulgaris (GenBank EF101866), (b) M. truncatula (GenBank AC124609.20), and (c) a subset of P. trichocarpa (NC_008472.1). Genes are indicated by boxes: NPR1 (gray box); CaMP encoding a calmodulin-binding protein (black box); specifying a CK1PK-class protein kinase (white box). Arrows indicate the direction of transcription. The gray bars indicating the distances in $\mathrm{Kb}$ between genes are from the end exon of one gene to the end exon of the next gene. The length of the DNA regions under comparison is indicated in base pairs. As of this writing, the TIGR annotated NPR1 region for M. truncatula is a draft; shown is only about one half of a BAC sequence since there was a gap in the sequence near the center.

(Table 2) clearly indicates that they are orthologous gene pairs with shared functions.

The three orthologous gene pairs NPR1, CaMP, and $C K 1 P K$ are colinear, that is, with both the same gene order and direction of transcription, in B. vulgaris, M. truncatula, and $P$. trichocarpa (Figure 2). In particular, in the comparison of presumptive orthologous gene pairs in B. vulgaris and M. truncatula, the NPR1, CaMP, and CK1PK genes encode proteins that have amino acid identities of about $60 \%, 57 \%$, and $67 \%$, and that exhibit amino acid similarities of about $74 \%, 68 \%$, and $77 \%$, respectively (Table 2 ). Similarly, in the comparison of presumptive orthologous gene pairs in $B$. vulgaris and $P$. trichocarpa, the three orthologous gene pairs, (NPR1, CaMP, and CK1PK), produce protein products with amino acid identities of about $65 \%, 56 \%$, and $75 \%$ and amino acid similarities of about $79 \%, 68 \%$, and $82 \%$, respectively (Table 2). Finally, comparison of the amino acid identities and similarities of the predicted products of the NPR1, CaMP, and CK1PK genes in P. trichocarpa and $M$. truncatula (Table 2) indicates conserved orthologous gene pairs.

As mentioned above, about $2 \mathrm{~Kb}$ downstream of NPR 1 in B. vulgaris, a fourth core plant gene (Figure 2) was predicted to produce a protein product with moderately high similarity to that produced by the embryonically expressed heat shock factor (HSF) A9 gene in sunflower. Microcolinearity of this particular HSF gene with NPR1 did not occur in $M$. truncatula. On the other hand, NPR1 in P. trichocarpa is separated from an HSF gene by only $1 \mathrm{~Kb}$, but it encodes a protein that has only about $39 \%$ amino acid identity and $54 \%$ amino acid similarity with the protein encoded by the HSF gene adjacent to the NPR1 gene in B. vulgaris. Thus, the structure and function of the respective HSF proteins is not as highly conserved as were the three other colinear core plant genes in poplar and sugarbeet.
Comparison of the Arabidopsis NPR1 region with those of M. truncatula, P. trichocarpa, and B. vulgaris revealed that Arabidopsis lacks conserved microsynteny of CaMP and $C K 1 P K$ genes with NPR1. In O. sativa L., the NPR1 genomic region has a gene encoding a calmodulin-binding protein, but it is transcribed in the same direction as NPR1 rather than in opposing directions as in B. vulgaris and $M$. truncatula; also the CK1PK gene, most proximal to NPR1 in O. sativa $L_{\text {., }}$ is greater than $250 \mathrm{~Kb}$ away (not shown). Thus, microsynteny, as in three out of four eudicot species, did not occur in $O$. sativa $L$., perhaps not unexpectedly as it is a monocot.

The six other noncore genes were retrotransposon or DNA transposon ones. Four putative genes were predicted to encode proteins with strong BLAST hits to retrotransposons or retrotransposon-like genes. An integrase gene has four exons and, from amino acid positions 750 to 900, this ORF encodes an rve core domain $\left(E=3 \mathrm{e}^{-30}\right)$, being a characteristic of integrases. With the integrase gene and the downstream putative gene $\mathrm{Hp} 3$ being combined, both share about 98\% nucleotide identity (BLASTN) with highly related genes on a $9 \mathrm{~Kb}$ DNA contig (GenBank accession ABD83280) of BAC62 from sugarbeet chromosome 9 [26]. A putative reverse transcriptase gene about $5 \mathrm{~Kb}$ downstream of $\mathrm{Hp} 3$ consists of a single exon encoding a polyprotein with $\mathrm{Rvt2}$ RNA-dependent DNA polymerase domain $\left(E=7 \mathrm{e}^{-24}\right)$, an rve integrase domain $\left(E=6 \mathrm{e}^{-21}\right)$, and a poorly conserved $\left(E=8 \mathrm{e}^{-10}\right)$ gag capsid-like protein domain.

Coe1, previously identified by our group using LTR_ STRUC analysis as a novel composite of class I and class II elements [25], has three genes. Briefly, one gene has a single exon ORF1 (Table 1), encoding a retroelement-like protein. ORF1 is about $2 \mathrm{~Kb}$ upstream of a gene that encodes a DNA transposase since its predicted product produced many significant BLAST alignments with DNA transposases, with 
TABLE 2: Percent amino acid identity and similarity exhibited by amino acid alignments of the conserved products of orthologous gene pairs.

\begin{tabular}{lccc}
\hline $\begin{array}{l}\text { Orthologous } \\
\text { gene pair }\end{array}$ & B. vulgaris NPR1 & B. vulgaris CaMP & B. vulgaris CK1PK \\
Identity/similarity & $60 \% / 74 \%$ & $57 \% / 68 \%$ & Identity/similarity \\
\hline Medicago homologs & $65 \% / 79 \%$ & $56 \% / 68 \%$ & $67 \% / 77 \%$ \\
Populus homologs & Medicago NPR1 & Medicago CaMP & $75 \% / 82 \%$ \\
\hline & Identity/similarity & Identity/similarity & Medicago CK1PK \\
& $62 \% / 75 \%$ & $60 \% / 71 \%$ & Identity/similarity \\
\hline Populus homologs & & $69 \% / 78 \%$ \\
\hline
\end{tabular}

$E$-values equal to 0 . The very highly conserved transposase family tnp2 domain $\left(E=1 \mathrm{e}^{-94}\right)$ occurs in amino acid residue positions $200-400$ of the predicted protein. Coel's transposase gene is also flanked by ORF2, a pseudogene of a copia-like integrase, and all three genes are within LTRs [7]. ORF2 is $2 \mathrm{~Kb}$ downstream of the tnp2-class DNA transposase gene, and its predicted product showed, by BLAST analysis, a significant alignment with several putative retrotransposon proteins from wine grape and rice; for example, it aligned to $O$. sativa's putative Ty1-copia subclass retrotransposon (accession ABA95677) with $E$-value of $\leq \mathrm{e}^{-50}, 38 \%$ amino acid identity, and 49\% amino acid similarity.

Downstream of the gene encoding a calmodulin-binding protein, a putative reverse transcriptase gene encoded a predicted protein with only a moderate BLAST alignment $\left(E=3 \mathrm{e}^{-37}\right)$ to a reverse transcriptase $R v t-2$ domain found in a novel retrotransposon-like TIR-NBS-LRR-type disease resistance protein in $P$. trichocarpa Torr. \& Gray (Table 1), and the similarity corresponds to a shared $R v t-2-$ type domain.

In M. truncatula, a TIR-NBS-LRR-type resistance gene occurs downstream of NPR1 (not shown). The predicted product of the M. truncatula resistance gene analogue (RGA) near NPR1 has 39\% amino acid identity and 59\% amino acid similarity with the Gro1-4 nematode resistance gene of Solanum tuberosum (GenBank accession AY196152.1).

Comparative sequence analysis and gene annotation in B. vulgaris, M. truncatula, and P. trichocarpa revealed that these three divergent species of eudicots exhibit conserved microsynteny of genes that encode the centrally important disease resistance priming NPR1, a signal-peptide calmodulin-binding protein (CaMP), and a CK1-class dualspecificity protein kinase (CK1PK). The orthologs occur in the same order and with the same direction of transcription.

\section{DISCUSSION}

In this study, comparison of orthologous NPR1 gene regions of B. vulgaris, M. truncatula, and P. trichocarpa revealed for the first time conserved microsynteny of the defense-priming NPR1 gene with a CaMP gene, encoding a calmodulinbinding protein, and with a $C K 1 P K$ gene, specifying a CK1class dual-specificity protein kinase.

Calmodulin-binding proteins in plants are very diverse, exhibit various motifs, and perform a correspondingly wide variety of functions [27-30]. For example, an Arabidopsis ethylene-upregulated calmodulin-binding protein triggers senescence and death [31].

Calcium and calmodulin mediate a complex signal transduction network in plants through protein kinases (PKs), and some PKs are unique to plants [32]. They are literal "hubs" of sensory perception and signal transduction. Examples include a calmodulin-binding PK in Arabidopsis that negatively regulates tolerance to osmotic stress [32] and a calmodulin-binding PK in tobacco (Nicotiana tabacum L.), involved with negative regulation of flowering [33].

It seems reasonable to hypothesize that the CaMP gene product, which is a chloroplast-targeted, signal-peptide, calmodulin-binding transmembrane protein, could play a role in rapid activation of a defense cascade during either general stress or pathogen response. Just as the NPR1 gene is critical for disease resistance priming in plants, some calmodulin-binding proteins are pathogenesis-related. For example, de novo synthesis of a calmodulin-binding peptide with a DNA-binding domain at the N-terminus is induced by ethylene formed by the plant in response to wounding and/or infection [34]; also in Arabidopsis, another calmodulin-binding protein is pathogenesis-related as well [35]. Moreover, in Glycine max L. (soybean), specific calmodulin isoforms are required for the expression of pathogen-induced proteins upregulated by the NPR1 disease resistance control gene [36]. Conserved microsynteny of CK1PK, CaMP, and NPR1 genes, discovered in three out of four eudicot species examined, could be hypothesized to suggest that their protein products play essential cellular roles related to plant defense response.

We propose a new hypothesis that conserved gene microsynteny of certain core plant genes in eudicots may correlate with either similar subcellular localization or with similar function. Either possibility for the protein products of the three core plant genes herein described is plausible. Activated monomeric NPR1 functions in the plant nucleus, where CK1PKs are also localized. CK1s are believed to control circadian rhythm [37] and chromosome partitioning during meiotic cell division [38] in all eukarotic cells. It should be noted that in $O$. sativa a novel family of dualspecificity PKs is involved in controlling the plant responses to biotic as well as abiotic stresses [39]. Based on available literature $[30,31,39]$, the $C K 1 P K$ gene localized near $N P R 1$ in certain eudicots may play a role in controlling the expression of stress-responsive genes in plants.

A total of 11 retrotransposons (RTs), DNA transposons, and hypothetical genes lie within the approximately $80 \mathrm{~Kb}$ 
stretch of DNA between the NPR1 and CaMP genes in $B$. vulgaris; therefore, we conclude that this region is rich in repetitive elements, and several insertions of mobile genetic elements have occurred during evolutionary divergence (Kuykendall et al., unpublished). ORFs, originating from either retrotransposons or viruses, from DNA transposons and other repetitive elements need not be considered disruptive of the conservation of colinearity when the core genes nevertheless occur in physical proximity, in the same order, and are transcribed in the same relative direction. Fortunately, our approach was not to be dissuaded by the repetitive elements that occur between NPR1 and $C a M P$ in B. vulgaris comparing the orthologous regions of $M$. truncatula and $P$. trichocarpa, and thus we discovered conserved microsynteny for orthologous NPR1, CaMP, and $C K 1 P K$ genes in these three eudicot species. This conserved microsynteny of NPR1, CaMP, and CK1PK in B. vulgaris, $M$. truncatula, and $P$. trichocarpa likely has an evolutionary basis through a yet undefined selective advantage.

The observed conservation of microsynteny of the three core plant genes could be hypothesized to result, in part, from positive natural selection for physical proximity. Hypothetically, close physical linkage may facilitate coordinated expression of genes critical in certain controlling nuclear events, such as those for which the protein products of CK1PK and NPR1 are known, or are responsible, either directly or indirectly, for the initiation of a dynamic signal transduction cascade as can be hypothesized for the predicted product of CaMP.

Reverse transcriptase (RT) PCR expression studies will be useful in determining whether the CaMP or CK1PK is upregulated in response to the administration of factors which induce the production of either pathogenesis-related or stress-related proteins. Such studies will hopefully allow one to determine whether the chloroplast-targeted signalpeptide calmodulin-binding protein gene $(C a M P)$ or the nuclearly localized casein kinase 1 protein kinase gene $(C K 1 P K)$ may play a role(s) in pathogen and/or stress response.

In summary, in addition to NPR1 gene and the CaMP and $C K 1 P K$ genes herein described for the first time, the $130 \mathrm{~Kb}$ NPR1-carrying B. vulgaris genomic DNA segment has 14 other features predicted by gene finders trained with Arabidopsis. Whereas seven ORFs produce predicted proteins with probable functions that can be deduced from BLAST analysis, seven other ORFs had predicted protein products without any known function.

It is also interesting to note that an HSF gene is located just $2 \mathrm{~Kb}$ downstream of NPR1 in sugarbeet, and the predicted product of this gene is a DNA-binding HSF protein similar to that specified by the HSFA9 gene that controls early leaf morphogenesis in sunflower [24].

We conclude that conserved microsynteny of NPR1, $C a M P$, and $C K 1 P K$ in three eudicot species suggests strong positive natural selection for the maintenance of physical linkage of these particular genes whose vital protein products either control specific nuclear events or are involved in signal transduction.

\section{ACKNOWLEDGMENTS}

The authors gratefully acknowledge the generous advice and help of Dr. J. Mitch McGrath, Michigan State University, USDA-ARS (East Lansing, Mich, USA), and of Dr. Robert E. Davis, Research Leader at Molecular Plant Pathology Lab, Wallace Agricultural Research Center, USDA-ARS (Beltsville, Md, USA). They also thank Kenneth Trimmer and Tatsiana Kurushko for helpful suggestions on the manuscript. This research was made possible in part by the support of the Beet Sugar Development Foundation (Denver, Colo, USA). Mention of a particular product is intended only to allow for precise reproduction of the reported findings, and is not intended to endorse any particular product to the exclusion of others, which may also be suitable.

\section{REFERENCES}

[1] X. Dong, "NPR1, all things considered," Current Opinion in Plant Biology, vol. 7, no. 5, pp. 547-552, 2004.

[2] Z. Mou, W. Fan, and X. Dong, "Inducers of plant systemic acquired resistance regulate NPR1 function through redox changes," Cell, vol. 113, no. 7, pp. 935-944, 2003.

[3] H. Cao, X. Li, and X. Dong, "Generation of broad-spectrum disease resistance by overexpression of an essential regulatory gene in systemic acquired resistance," Proceedings of the National Academy of Sciences of the United States of America, vol. 95, no. 11, pp. 6531-6536, 1998.

[4] L. Friedrich, K. Lawton, R. Dietrich, M. Willits, R. Cade, and J. Ryals, "NIM1 overexpression in Arabidopsis potentiates plant disease resistance and results in enhanced effectiveness of fungicides," Molecular Plant-Microbe Interactions, vol. 14, no. 9, pp. 1114-1124, 2001.

[5] M. Chern, H. A. Fitzgerald, P. E. Canlas, D. A. Navarre, and P. C. Ronald, "Overexpression of a rice NPR1 homolog leads to constitutive activation of defense response and hypersensitivity to light," Molecular Plant-Microbe Interactions, vol. 18, no. 6, pp. 511-520, 2005.

[6] W.-C. Lin, C.-F. Lu, J.-W. Wu, et al., "Transgenic tomato plants expressing the Arabidopsis NPR1 gene display enhanced resistance to a spectrum of fungal and bacterial diseases," Transgenic Research, vol. 13, no. 6, pp. 567-581, 2004.

[7] R. Makandar, J. S. Essig, M. A. Schapaugh, H. N. Trick, and J. Shah, "Genetically engineered resistance to fusarium head blight in wheat by expression of Arabidopsis NPR1," Molecular Plant-Microbe Interactions, vol. 19, no. 2, pp. 123-129, 2006.

[8] J. M. McGrath, R. S. Shaw, B. G. de los Reyes, and J. J. Weiland, "Construction of a sugar beet BAC library from a hybrid with diverse traits," Plant Molecular Biology Reporter, vol. 22, no. 1, pp. 23-28, 2004.

[9] D. Kuykendall, T. Murphy, J. Shao, and J. M. McGrath, "Nucleotide sequence analyses of a sugar beet genomic NPR1class disease resistance gene," Journal of Sugar Beet Research, vol. 44, pp. 35-49, 2007.

[10] L. K. Mosavi, T. J. Cammett, D. C. Desrosiers, and Z.-Y. Peng, "The ankyrin repeat as molecular architecture for protein recognition," Protein Science, vol. 13, no. 6, pp. 1435-1448, 2004.

[11] U. Conrath, G. J. M. Beckers, V. Flors, et al., "Priming: getting ready for battle," Molecular Plant-Microbe Interactions, vol. 19, no. 10, pp. 1062-1071, 2006. 
[12] R. L. Bargabus-Larson and B. J. Jacobsen, "Biocontrol elicited systemic resistance in sugarbeet is salicylic acid independent and NPR1 dependent," Journal of Sugar Beet Research, vol. 44, pp. 17-33, 2007.

[13] H.-M. Ku, T. Vision, J. Liu, and S. D. Tanksley, "Comparing sequenced segments of the tomato and Arabidopsis genomes: large-scale duplication followed by selective gene loss creates a network of synteny," Proceedings of the National Academy of Sciences of the United States of America, vol. 97, no. 16, pp. 9121-9126, 2000.

[14] I. Dominguez, E. Graziano, C. Gebhardt, et al., "Plant genome archaeology: evidence for conserved ancestral chromosome segments in dicotyledonous plant species," Plant Biotechnology Journal, vol. 1, no. 2, pp. 91-99, 2003.

[15] G. E. Coe and G. J. Hogaboam, "Registration of US H20 sugarbeet," Crop Science, vol. 11, no. 6, p. 942, 1971.

[16] A. C. J. Frijters, Z. Zhang, M. van Damme, G.-L. Wang, P. C. Ronald, and R. W. Michelmore, "Construction of a bacterial artificial chromosome library containing large EcoRI and HindIII genomic fragments of lettuce," Theoretical and Applied Genetics, vol. 94, no. 3-4, pp. 390-399, 1997.

[17] S. F. Altschul, W. Gish, W. Miller, E. W. Myers, and D. J. Lipman, "Basic local alignment search tool," Journal of Molecular Biology, vol. 215, no. 3, pp. 403-410, 1990.

[18] M. Borodovsky and J. McIninch, "GENMARK: parallel gene recognition for both DNA strands," Computers and Chemistry, vol. 17, no. 2, pp. 123-133, 1993.

[19] A. V. Lukashin and M. Borodovsky, "GeneMark.hmm: new solutions for gene finding," Nucleic Acids Research, vol. 26, no. 4, pp. 1107-1115, 1998.

[20] J. Schultz, F. Milpetz, P. Bork, and C. P. Ponting, "SMART, a simple modular architecture research tool: identification of signaling domains," Proceedings of the National Academy of Sciences of the United States of America, vol. 95, no. 11, pp. 5857-5864, 1998.

[21] G. A. Tuskan, S. DiFazio, S. Jansson, et al., "The genome of black cottonwood, Populus trichocarpa (Torr. \& Gray)," Science, vol. 313, no. 5793, pp. 1596-1604, 2006.

[22] S. B. Cannon, J. A. Crow, M. L. Heuer, et al., "Databases and information integration for the Medicago truncatula genome and transcriptome," Plant Physiology, vol. 138, no. 1, pp. 3846, 2005.

[23] N. D. Young, S. B. Cannon, S. Sato, et al., "Sequencing the genespaces of Medicago truncatula and Lotus japonicus," Plant Physiology, vol. 137, no. 4, pp. 1174-1181, 2005.

[24] C. Almoguera, A. Rojas, J. Díaz-Martín, P. Prieto-Dapena, R. Carranco, and J. Jordano, "A seed-specific heat-shock transcription factor involved in developmental regulation during embryogenesis in sunflower," Journal of Biological Chemistry, vol. 277, no. 46, pp. 43866-43872, 2002.

[25] D. Kuykendall, J. Shao, and K. Trimmer, "Coe1 in Beta vulgaris L. has a Tnp2-domain DNA transposase gene within putative LTRs and other retroelement-like features," International Journal of Plant Genomics, vol. 2008, Article ID 360874, 7 pages, 2008.

[26] D. Schulte, D. Cai, M. Kleine, L. Fan, S. Wang, and C. Jung, "A complete physical map of a wild beet (Beta procumbens) translocation in sugar beet," Molecular Genetics and Genomics, vol. 275, no. 5, pp. 504-511, 2006.

[27] A. R. Rhoads and F. Friedberg, "Sequence motifs for calmodulin recognition," The FASEB Journal, vol. 11, no. 5, pp. 331340, 1997.
[28] M. Bähler and A. Rhoads, "Calmodulin signaling via the IQ motif," FEBS Letters, vol. 513, no. 1, pp. 107-113, 2002.

[29] V. S. Reddy, G. S. Ali, and A. S. N. Reddy, "Genes encoding calmodulin-binding proteins in the Arabidopsis genome," Journal of Biological Chemistry, vol. 277, no. 12, pp. 98409852, 2002.

[30] M. Charpenteau, K. Jaworski, B. C. Ramirez, A. Tretyn, R. Ranjeva, and B. Ranty, "A receptor-like kinase from Arabidopsis thaliana is a calmodulin-binding protein," Biochemical Journal, vol. 379, no. 3, pp. 841-848, 2004.

[31] T. Yang and B. W. Poovaiah, "An early ethylene up-regulated gene encoding a calmodulin-binding protein involved in plant senescence and death," Journal of Biological Chemistry, vol. 275, no. 49, pp. 38467-38473, 2000.

[32] E. Perruc, M. Charpenteau, B. C. Ramirez, et al., "A novel calmodulin-binding protein functions as a negative regulator of osmotic stress tolerance in Arabidopsis thaliana seedlings," The Plant Journal, vol. 38, no. 3, pp. 410-420, 2004.

[33] W. Hua, L. Zhang, S. Liang, R. L. Jones, and Y.-T. Lu, "A tobacco calcium/calmodulin-binding protein kinase functions as a negative regulator of flowering," Journal of Biological Chemistry, vol. 279, no. 30, pp. 31483-31494, 2004.

[34] A. S. N. Reddy, V. S. Reddy, and M. Golovkin, "A calmodulin binding protein from Arabidopsis is induced by ethylene and contains a DNA-binding motif," Biochemical and Biophysical Research Communications, vol. 279, no. 3, pp. 762-769, 2000.

[35] V. S. Reddy, G. S. Ali, and A. S. N. Reddy, "Characterization of a pathogen-induced calmodulin-binding protein: mapping of four $\mathrm{Ca}^{2+}$-dependent calmodulin-binding domains," Plant Molecular Biology, vol. 52, no. 1, pp. 143-159, 2003.

[36] C. Y. Park, W. D. Heo, J. H. Yoo, et al., "Pathogenesis-related gene expression by specific calmodulin isoforms is dependent on NIM1, a key regulator of systemic acquired resistance," Molecules and Cells, vol. 18, no. 2, pp. 207-213, 2004.

[37] B. Kloss, J. L. Price, L. Saez, et al., "The Drosophila clock gene double-time encodes a protein closely related to human casein kinase I $\varepsilon, "$ Cell, vol. 94, no. 1, pp. 97-107, 1998.

[38] M. Petronczki, J. Matos, S. Mori, et al., "Monopolar attachment of sister kinetochores at meiosis I requires casein kinase 1," Cell, vol. 126, no. 6, pp. 1049-1064, 2006.

[39] Z. Gu, J. Wang, J. Huang, and H. Zhang, "Cloning and characterization of a novel rice gene family encoding putative dual-specificity protein kinases, involved in plant responses to abiotic and biotic stresses," Plant Science, vol. 169, no. 3, pp. 470-477, 2005. 

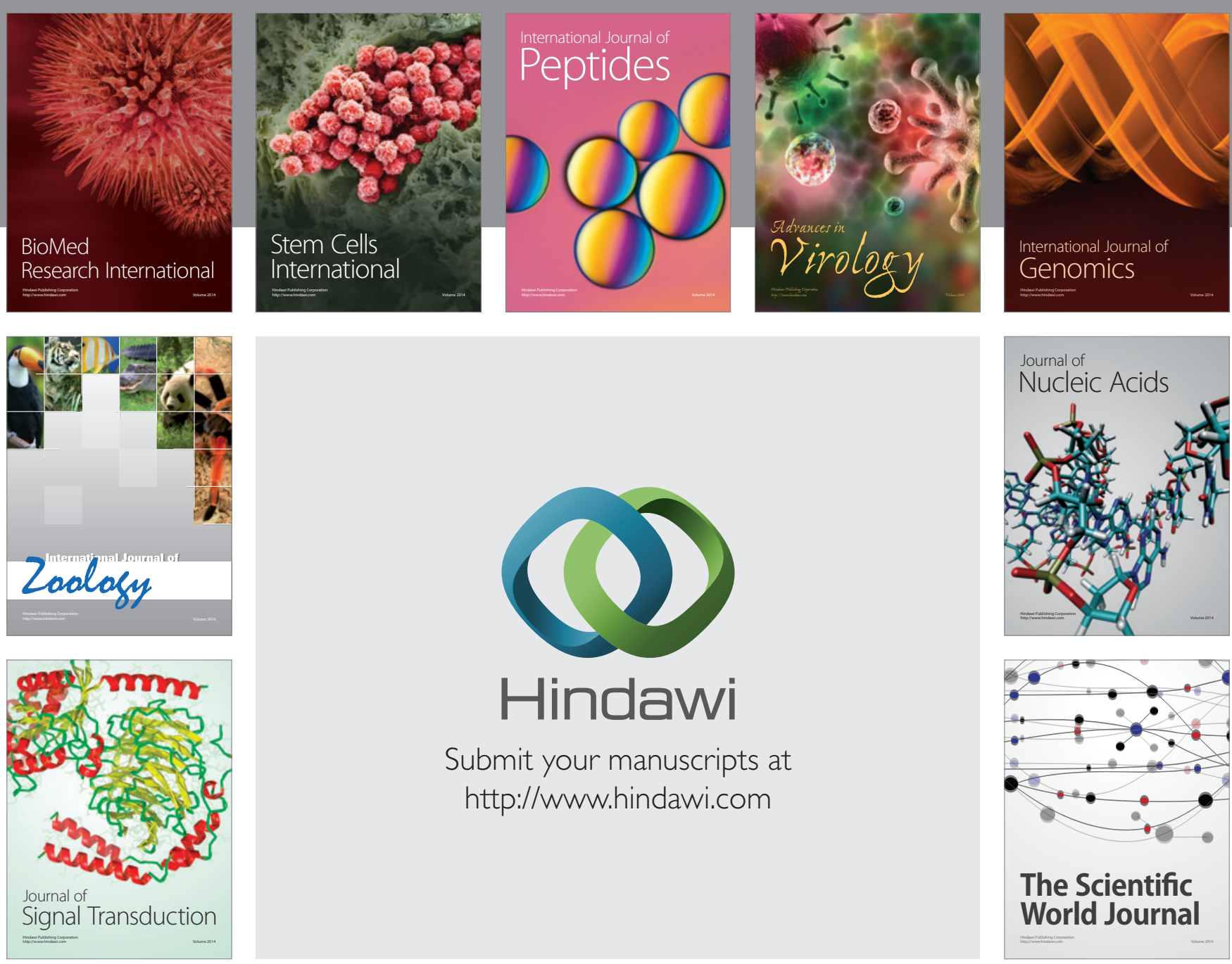

Submit your manuscripts at

http://www.hindawi.com
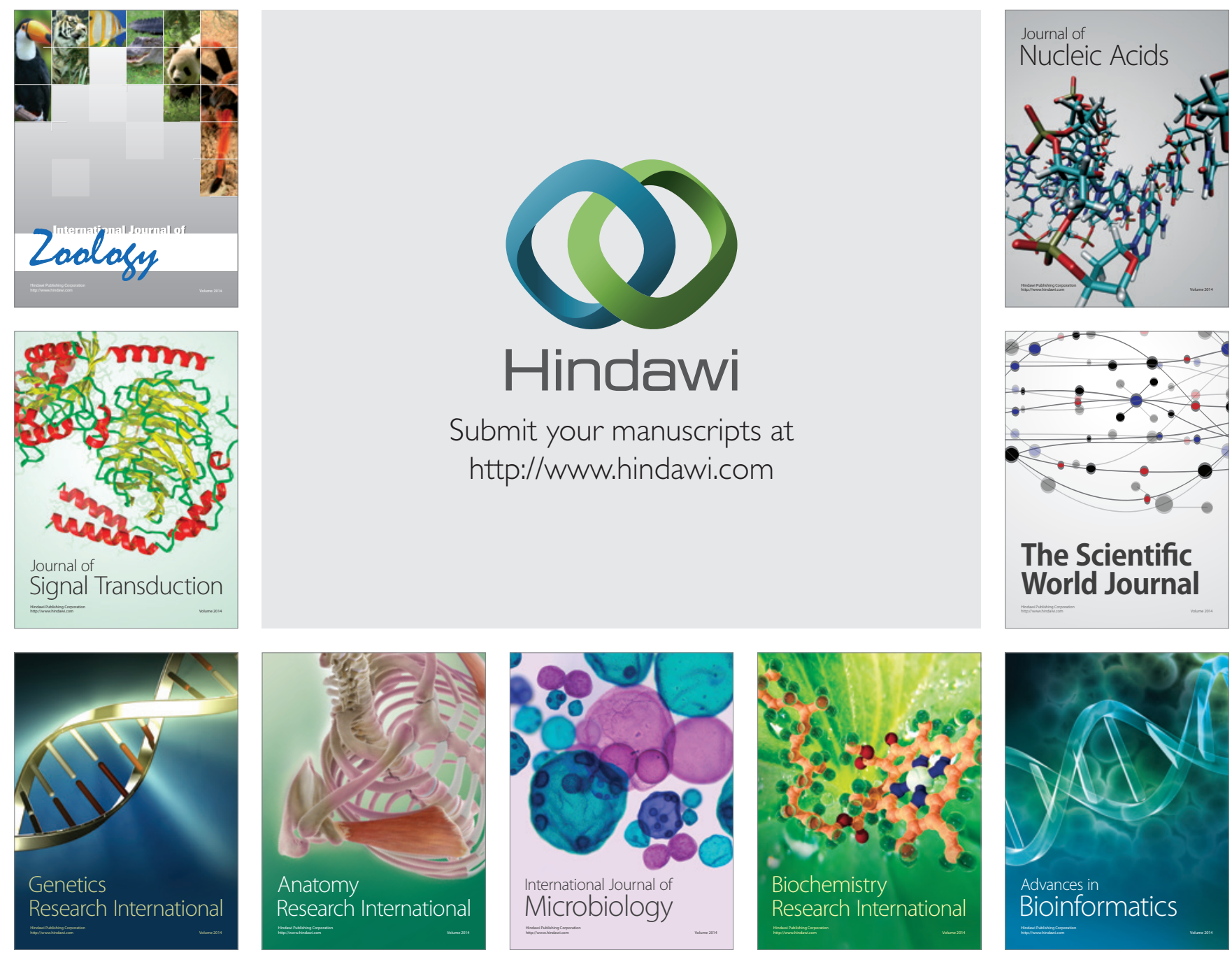

The Scientific World Journal
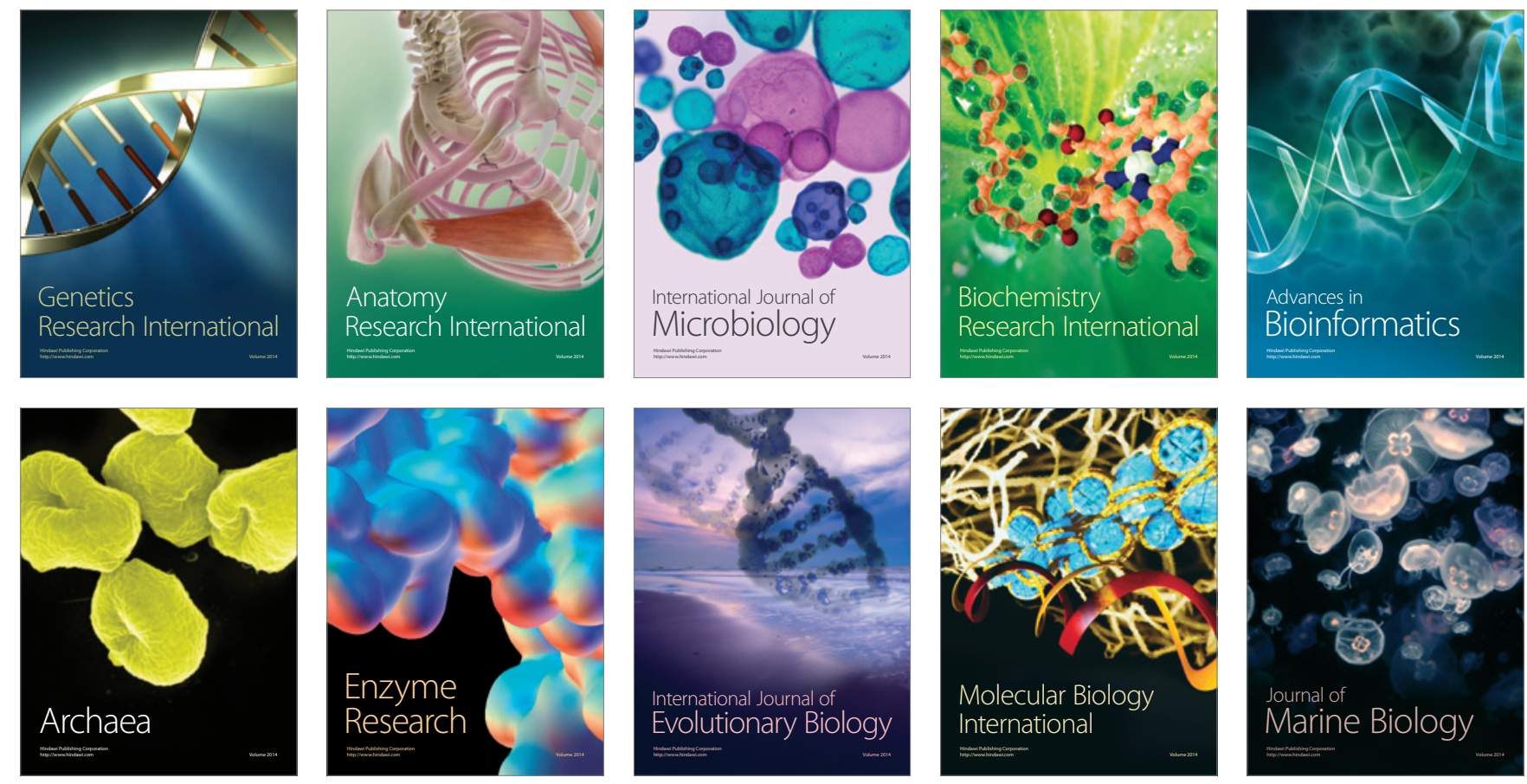